\title{
LÉGISLATION
}

\section{Cahier des Charges pour la concession par l'Etat d'une distribution publique d'énergie électrique ${ }^{(*)}$}

(Non applicable à une concession de distribution aux services publics)

N. B. - Les mots ou phrases en italiques peuvent ètre maintenus ou rayés, au choix de l'autorité concédante.

Article premier. - Service concédé. - La présente concession a pour objet la distribution publique de l'énergie électrique

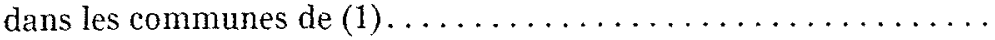

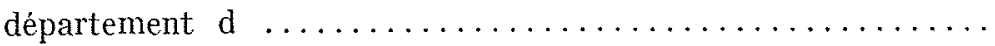

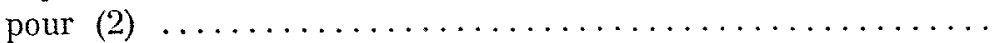
La concession ne comprend pas la fourniture de l'énergie électrique pour force motrice aux entreprises de transport en commun et aux établissements ou services ci-après énumérés :

Ces entreprises ou établissements peuvent toutefois être desservis par le concessionnaire dans les conditions prévues à l'article 3 ci-après.

Art. 2. - Droit d'utiliser les voies publiques. - La concession confère au concessionnaire le droit d'établir et d'entretenir, dans le périmètre de sa concession, soit au-dessus soit au-dessous des voies publiques et de leurs dépendances, tous ouvrages ou canalisations destinés à la distribution de l'énergie électrique, en se conformant aux conditions du présent cahier des charges, aux règlements de voirie et aux décrets ou arrêtés intervenus en exécution de la loi du 15 juin 1906.

Le concessionnaire ne pourra réclamer aucune indemnité pour le déplacement ou la modification des ouvrages établis par lui sur les voies publiques, lorsque ces changements sont requis par l'autorité compétente pour un motif de sécurité publique ou dans l'intérêt de la voirie.

ART. 3. - Ltilisation accessoire des ouvrages et canalisations. Le concessionnaire est autorisé à faire usage des ouvrages et canalisations établis en vertu de la présente concession pour desservir les entreprises de transport en commun, les établissements ou services énumérés à l'article $1^{\mathrm{er}}$ ci-dessus et d'une manière générale toutes entreprises situées hors de la concession, à la condition expresse qu'il n'en résulte aucune entrave au bon fonctionnement de la distribution et que toutes les obligations du cahier des charges soient remplies.

ART. 4. - Origine du matériel. - Le matériel nécessaire à l'établissement des ouvrages de la distribution pourra être em-

(*) Ce cahier des charges, publié au Journal officiel du 10 janvier 1928 , p. 1674 à 1679 , en annexe au décret du 17 janvier 1928, remplace le cahier des charges annexé au décret du 28 juin 1921. (Extıait d z la Revue générale de l'Electricité, Mars 1928).

(1) Indiquer les communes ou parties de communes sur lesquelles porte la concession.

(2) Suivant que la concession comporte la distribution de l'énergie électrique en vue de l'éclairage seul, en vue de tous usages ou en vue de tous usages autres que l'éclairage, d'une des trois formules suivantes sera employée :

L'éclairage public ou privé ;

Tous usages ;

Tous usages autres que l'éclairage public ou privé. ployé el mis en ceuvre par les concessiomnares, sans aulorisation préalable, s'il provient de sociétés ou constructeurs français et s'il a été fabriqué en lirance.

Si le concessiomaire se irouve dans l'impossibilité de se le procurer en tout ou en partie, dans des conditions normales satisfaisantes de temps, de prix et de qualité, il pourra recourir aux fournisseurs étrangers, avec l'autorisation du ministre des Travaux publics.

Dans lous les cas, il en sera donné avis au service de contrôle.

L'approbation ou le défaul d'approbation administrative n'aura pour effel ni d'engager la responsabilité de l'administration, ni de dégager le concessionnaire des conséquences que pourrait avoir l'exécution des travaux, l'imperfection des dispositions prévues ou le fonctionnement de l'ouvrage.

ArT. 5. - Ounrages à établir pour la distribution. - Le concessionnaire sera tenu d'élablir les canalisations, sous-stations, postes de transformation, etc., nécessaires à la distribution, représentés sur le plan annexé au présent cahier des charges (1).

La consistance des installations définies sur le plan annexe pourra ètre modifiée par un accord entre l'autorilé concédante et le concessionnaire.

Le réseau sera desservi au moven d'un ou plusieurs postes d'alimentation qui feronl partie integrante de la distribution et seront situés à l'intérieur de son périmètre.

Les ouvrages destinés à la produclion de l'énergie et à son transport jusqu'à chacun de ses postes ne feront pas partie de la présente concession.

Toutefois, le concessionnaire sera tenu de construire et de maintenir en bon état de service une (ou plusieurs) usine rgénératrice d'une puissance totale d'au moins......... kilowatts. Cette (ou ces) usine, ainsi que les ouvrages la (ou les) reliant au réseau de distribution feront partie de la concession (2).

L'Etat remet au concessionnaire, qui accepte, l'ensemble des installations (immeubles. canalisations, oubrages, matériels et

(1) Cet alinéa sera supprimé lorsque le concessionnaire n'a à établir aucune partie du réseau initial. Au lieu de déterminer par un plan les lignes constituant le réseau à établir par le concessionnaire, l'Etat peut imposer l'établissement d'une longueur donnée de canalisations principales.

Dans ce cas, les mots "représentés sur le plan annexé au présent cahier des charges "du premier alinéa, doivent être supprimés, ainsi que le deuxième alinéa, qui sera remplacé par l'alinéa suivant :

"Il devra établir au moins ..... mètres de canalisations principales. "

(2) L'Etat peut exiger que les usines dépendant de la concession soient en état de produire toute l'énergie nécessaire à la distribution; dans ce cas, les deuxième, troisième et quatrième alinéas de l'article 5 doivent être supprimés et le premier alinéa complété par les mots suivants : "ainsi que les ouvrages destinés à la production de l'énergic et à son transport juscuu'au réseau, lesquels feront partie intégrante de la concession ". 
appareils) lui appartenanl; représentés sur le plan annexé au présent cahier des charges ol accompagné d'un inventaire desdiles installations (1).

Les installations ainsi remises par l'Eral au concessionnaire, pour la durée de la concession, font partie intégranle de la distribution; en particulier l'entretien el le renouvellement en incombent au concessionnaire.

En contre-partie de l'usage des ouvrages mis à sa disposilion par l'Etal, le concessionnaire lui versera une redevance spériale déterminée comme il suil: (2)

ArT. 6. - Délais d'exécution. Les projels d'exécution des ouvrages el des lignes désignées sur le plan annexé au présent cahier des charges devront être présentés par le concessionnaire dans le délai de (3)..... mois à partir de l'approbation définitive de la concession (4).

Les travaux seront commencés dans le délai de......... $\ldots \ldots \ldots \ldots \ldots$, à dater de l'approbation des projets el poursuivis sans interruption, de manière à être achevés dans le délai de............

ArT. 7. - Propriété des installations. -- Le concessiomnaire sera lenu d'acquérir les machines et l'outillage nécessaires à la distribution.

Il pourra, à son choix, soil acquérir les terrains et établir à ses frais les constructions affectées au service de la distribution, soil les prendre en location.

Toutefois, il sera tenu d'acquérir en toute propriété et de construire les (5).......

Pour établissement des ouvrages, l'Etat s'engage it mettre à la disposition du concessionnaire moyennant $(6) . \ldots \ldots \ldots$

Les baux ou contrats relatifs à toules les locations d'immeubles seront communiqués au préfet ; ils devront comporter une clause réservanl expressément à l'Etat la faculté de se substituer au concessionnaire en cas de rachat ou de déchéance. II en sera de même pour tous les contrats de fourniture d'énergie, si le concessionnaire achète le courant.

ART. 8. - Nalure et mode de protection du courant. - L'Etat aura le droit, à toute époque, de faire mettre à la disposition du concessionnaire de l'énergie réservè aux bornes d'une usine hydraulique concédée.

(1) Alinéa applicable dans le cas où l'Etat est propriétaire, au moment de l'institution de la concession, d'un réseau de distribution déjà existant.

(2) Cette redevance pourra selon les modalités de la convention à intervenir ètre, soit fixe, soit fonction des recettes brutes, du nombre de kilowatts souscrits ou de kilowatts-heures distribués; elle peut varier suivant la tension et l'usage du courant. On peut également prévoir une combinaison de ces modalités.

(3) Ces ouvrages et ces lignes peuvent ètre séparés en réseaux d'urgences difféa entes pour lesquels ce délai aura des valeurs distinctes

(4) Dans le cas où au lieu de déterminer les canalisations constituant le réseau à établir immédiatement, l'article 5 impose l'établissement d'une longueur domnée de canalisations principales, le premier alinéa de l'article 6 doit ètre rédigé de la manière suivante : Le concessionnaire devra présenter dans le délai de ....... mois, à partir de l'approbation de la concession, le projet des ouvrages dont l'article 5 lui impose l'établissement. L'article 6 n'a pas à ètre inséré lorsque le concessiomnaire n’a à établir aucune partie du réseau initial.

(5) L'Etat peut imposer au concessiomnaire l'acquisition en loute propriété des inmmeubles destinés à l'établissement des usines de production et des sous-stations.

(6) L'Etat peut autoriser, par le cahier des charges, le concessionnaire à occuper dans des conditions déterminées les parties du domaine public dont il a la disposition.
Usines généralrices (1).

Sous-stations el postes de transformation (2).

Ar't. 9. - Tension el fréquence. -- Basse lension. -..- Lá Lension du courant distribué en basse tension aux abonnés est fixée à..

La lolérance maximum pour la variation de la lension en basse tension est de........ pour 100 en plus ou en moins pour l'éclairage, et de..... pour 100 en plus ou en moins pour tous autres usages (3).

Haute tension. - La tension du courant distribué en haute tension aux abonnés est fixée à.........

La valeur de la tension fixée dans chaque contrat d'abonnement en haute tension ne devra pas s'écarter de plus de..... . pour 100 en plus ou en moins des valeurs ci-dessus; la tension mesurée au point d'ulilisation en service normal ne devra pas elle-même s'écarter de plus de ........ pour 100 en plus ou en moins des valeurs fixées au contral d'abonnement.

Fréquence. - La fréquence du courant distribué est fixée à ....... périodes par seconde; elle ne doit pas varier de plus de ....... pour 100 en plus ou en moins de sa valeur normale (4).

Art. 10. - Canalisations. - Les canalisations souterraines seront placées directement dans le sol; toutefois, elles pourront, sur la demande du concessionnaire, être placées dans des galeries accessibles et devront l'être lor sque les services de voirie l'exigeront. Sauf aux traversées des chaussées, elles seront toujours sous les trottoirs, à moins d'unne autorisation spéciale.

A la traversée des chaussées, fondées sur bêtons et des voies de tramways, les dispositions nécessaires seront prises pour que le remplacement des canalisations soit possible sans ouverture de tranchée.

Les canalisations aériennes (5) .......

Branchements particuliers (6)

Art. 11. - Tarif maximum. - Les prix auxquels le concessionnaire est autorisé à vendre l'énergie électrique ne peuvent dépasser les maxima suivants (7) :

(1) Lorsque l'acte de concession prévoit la construction d'usines génératrices faisant partie intégrante de la concession, l'article 8 détermine les conditions d'établissement de ces usines.

(2) L'article 8 détermine également, s'il y a lieu, les conditions d'établissement des sous-stations et des postes de transformation.

(3) La tolérance de tension admise habituellement pour l'éclairage est de :

5 pour 100 en plus ou en moins pour les distributions urbaines d'au moins $4.000 \mathrm{kw}$.

7 pour 100 en plus ou en moins pour les distributions urbaines de moins de $4.000 \mathrm{kw}$.

10 pour 100 en plus ou en moins pour les distributions rurales.

(4) Cet alinéa ne s'applique qu'aux distributions de courant alternatif. La tolérance habituellement admise pour la fréquence ne dépasse pas 5 pour 100 .

(5) L'Etat peut interdire les canalisations aériennes : lorsqu'elles sont autorisées, il convient d'indiquer si les canalisations peuvent ètre aériennes dans toute l'étendue de la concession ou sinon dans quelles parties elle ne peuvent l'ètre.

L'Etat peut, en autorisant les canalisations aériennes, déterminer les conditions auxquelles sera soumis leur établissement.

(6) L'article 10 détermine, s'il y a lieu, les conditions auxquelles doivent satisfaire les branchements particuliers.

(7) Des tarifs différents peuvent être prévus, suivant la tension, la puissance, l'utilisation, l'usage du courant, l'horaire, les saisons, les sujétions spéciales acceptées par certains abonnés, la distance aux usines ou aux postes, etc.

La tarification peut consister, soit en une taxe de kilowattheure consommé, soit en une formule binome comportant un elé- 
Le tarif maximum de base s'entend, pour la basse lension, d'une situation économique conventionnellement caractérisée par la valeur 130 de l'index économique électrique bass" tension fixé périodiquement par le ministre des Tramaur publics, d'après les prix des houilles et de la main-d' auvre pour le département de..... .

Dans le cas oì la valeur de cet index s'écarterait par excés ou par défaut de plus de 10 o de la naleur caractérislique de 130 , il serait ajouté aux tarifs maxima de base (ou retranche de ces tarifs) un terme correctif donné par la formule (1):

Ce terme correctif sera appliqué (2).

Le tarif maximum de base s'entend, pour la haute tension, d'une situation économique conventionnellement caractérisée par la valeur de 100 de l'index économique électrique haute tension fixé périodiquement par le ministre des Travaux publics, d'après le prix des houilles et de la main-d'œuvre.

ment fonction de l'énergie consommée (élément énergie), soit cn un tarif de vente à forfait.

Dans les cas spéciaux où cela est nécessaire, la tarification peut comporter une garantie d'un certain nombre d'heures d'utilisation. Il importe alors de préciser le taux nécessairement réduit auquel seront payés les kilowatts-heures garantis mais non consommés, par exemple, en spécifiant que ces kilowatts-heures ne seront passibles que d'une certaine fraction de la taxe par kilowatt-heure et du terme co rectif.

La tarification peut tenir compte de l'utilisation ou de la consommation sur l'ensemble du réseau et prévoir d'après ces éléments une réduction soit des prix de l'énergie, soit du minimum d'utilisation.

L'énergie réactive peut faire l'objet d'une tarification en haute tension.

(1) Cette formule devra ètre établie dans chaque cas d'espèce de manière à suivre, le plus exactement possible, les répercussions sur le prix de revient des variations des charges diverses incombant au concessionnaire, main-d'œuvre, dépenses d'entretien, frais de combustibles, taux de l'intérèt, etc.), et en tenant compte également des conditions de production de l'énergie par une usine thermique ou hydraulique.

On pourra établir des formules différentes pour chaque élément de chacun des tarifs. Entre autres combinaisons, on pourra adopter des formules de la forme $T=n(i-130), i$ étant l'index économique électrique basse tension et $n$ un coefficient qui peut ètre différent pour chaque élément des tarifs et qui sera calculé d'après les conditions particulières de la distribution.

L'index économique électrique basse tension est défini de la manière suivante : c'est un nombre qui, pour représenter conventionnellement la situation économique, est calculé en ajoutant au prix de la tonne de houille ou en retranchant de ce prix un autre nombre $N$ qui varie lui-mème avec le prix de la main-d'œuvre.

Le prix de la tonne de houille est établi périodiquement par région, par le ministre des Travaux publics, d'après le cours et la qualité des combustibles.

Le nombre $N$ relatif à la main-d'œuvre se calcule comme il suit : pour chaque période, le ministre des Travaux publics constate la moyenne des salaires horaires des agents des entreprises de production et de distribution, directeurs exclus, pour l'ensemble du territoire et cette moyenne est comparée à la moyenne de l'année 1918.

S'il résulte de cette comparaison entre les moyennes constatées par convention de $t$. pour 100 , le nombre $N$ est pris égal par convention à $0.60 t$.

Si la moyenne est supérieure à celle de 1918 qui est prise pour base de comparaison, le nombre $N$, calculé comme il vient d'être dit, est ajouté au prix de la tonne de houille : si cette moyenne est inférieure à celle de 1918 , le nombre $N$ est retranché du prix de la tonne de houille. Pour la constatation de la moyenne, il est tenu compte de tous les appointements et salaires jusqu'au directeur exclusivement, avec tous les accessoires, tels que suppléments pour cherté de vie, indemnité de résidence, charges de retraites, etc.

(2) La période d'application de chaque index pourra, pour la vente en basse tension, être déterminé de manière à ne pas entrầner l'obligation de faire un appel ou une ristourne sur les consommations déjà facturées.
Dans le cas on la valeur de cel index s'écarterait par excès ou par défaut de plus de.... pour 1000 (1) de la valeur caractéristique de 100 il serail, pendant la période correspondante, ajouté aux tarifs maxima de base (ou retranché de ces larifs) un lerme correctif donné par la formule (2):

Revision des tarifs. - Pour maintenir les larifs en harmonie avec les variations des itronstances économiques da pays, les termes correctifs pourront ètre revisés à la demande, soil de l'Etal, soit du concessionnaire:

$1^{\circ}$ Si l'index économique électrique s'écarte de plus de ...... pour 1000 (3) de la valeur de cet index au moment de la dernière fixation des tarifs;

$2^{\circ}$ S'il est procédé à la revision des tarifs de base;

30 S'il est écoulé plus de ........ années, depuis la dernière fixation des tarifs.

Les tarifs maxima de base seront revisés sur la demande soit de l'Etat, soit du concessionnaire :

10 Si, par suite de l'élablissement d'une distribution nouvelle d'énergie aux services publics concédés par l'Elat, ou d'une usine hydraulique, le concessionnaire peut s'alimenter plus avantageusement au moyen de celle distribution ou de cetle usine ;

$2^{\circ}$ Si la distribution étant alimentée par une autre distribution aux services publics, concédés par l'Etat, les tarifs de cette concession seront revisés;

$3^{0}$ Si au cours de la concession, l'Etat fait mettre à la disposition du concessionnaire de l'énergie réservée aux bornes d'une usine hydraulique concédée;

$4^{\circ}$ Si la distribution étant alimentée en tout ou en partie par des réserves d'énergie aux bornes d'une usine hydraulique concédée, les tarifs de cette énergie aux bornes de l'usine seront revisés.

Dans les quatre cas ainsi prévus, la revision sera opérée en partant des tarifs maxima de base fixés par le présent article et en modifiant ces tarifs, pour tenir un compte équitable de la répercussion, sur le prix de revient moyen de l'énergie, des conditions nouvelles dans lesquelles la concession peut être alimentée.

Dans les premiers et troisième cas, il sera tenu compte des engagements antérieurement pris par le concessionnaire envers ses fournisseurs du courant.

En cas d'accord entre l'Etat et le concessionnaire, l'avenant portant soil revision du lerme correctif, soit fixation des nouveaux tarifs de base, ne sera définilif qu'après avoir été approuvés par la même autorité que le présent cahier des charges.

(1) Ce pourcentage ne devra pas être inférieur à 5 pour 100 ni supérieur à 10 pour 100 .

(2) Cette formule devra ètre établie, dans chaque cas d'espèce, de manière à suivre avec le plus d'exactitude possible, les répercussions sur le prix de revient des charges diverses incombant au concessionnaire (main-d'œuvre, dépenses d'entretien, frais de combustibles, taux de l'intérêt, etc.).

On pourra établir des formules différentes pour chaque élément de chacun des tarifs. Entre autres combinaisons, on pourra adopter des formules de la forme $I=n(i-100), i$ étant l'index économique électrique haute tension et $n$ un coefficient qui peut être différent pour chaque élément des tarifs et qui sera calculé d'après les conditions particulières de la distribution.

L'inde s économique électrique haute tension est déterminé de la même façon qu'il a été dit ci-dessus de l'index économique électricque haute tension, mais le nombre $N$ relatif à la maind'œu vre est pris égal $0,25 \mathrm{l}$ au lieu de 0,60 .

(3) Au maximum 50 pour 100. 
Si, dans les six mois, à compter de la date fixée pour la revision, un accord n'est pas intervenu, il sera procédé à cetle revision par une commission composée de trois menbres dont l'un sera désigné par le préfet ou le ministre des Travaux publics (1), un autre par le concessionnaire et le troisième par les deux premiers. Faute par ceux-ci de s'entendre dans le délai de quinze jours, la désignation du troisième membre sera faite par le président du Comilé d'Electricité sur une liste arrêtée par ce comité.

Les tarils revisés auront leur effel dans un délai de (2) . . . $\ldots \ldots \ldots \ldots \ldots$ à partir de la date de la demande de revision.

Abaissement des larifs en basse lension. - Si le concessionnaire abaisse pour certains abonnés les prix de vente de l'énergic à basse tension, avec ou sans conditions au-dessous des limites fixées par le tarif maximum prévu ci-dessus, il sera tenu de faire bénéficier des mêmes réductions lous les abonnés placés dans les mèmes conditions de puissance, d'horaire, d'utilisation, de consommation, de durée d'abomnement et de tarif maximum.

A cet effet, il devra établir et tenir constamment à jour un relevé de tous les abaissements consentis, avec mention des conditions auxquelles ils sont subordonnés. Un exemplaire de ce relevé sera déposé dans chacun des bureaux où peuvent ètre contractés des abonnements et tenu constamment à la disposition du public et des agents de contrôle.

ArT. 12. - Tarifs applicables aux services publics. - Les services publics de l'Etal, des départements et des communes, les établissements publics et les associations syndicales organisées par l'administration en vertu des lois des 16 septembre 1807 et 8 avril 1898 ou autorisées en conformité des lois des 21 juin 1865 et 22 décembre 1888 , bénéficieront, sur le tarif maximum prévu à l'article ci-dessus y compris le terme correctif, des réduclions suivantes (3) :

Les groupements agricoles d'utilité générale spécifiès dans le règlement d'administration publique prévu par l'article 10 de la loi du 16 octobre 1919, bénéficieront d'une réduction de ....... pour 100 .

Le concessionnaire sera tenu, sur la réquisition du ministre dre Travaux publics, de recevoir sur son réseau, dans la limite de capacité de ce dernier, l'énergie destinée à des services publics, établissements publics, associations syndicales et groupements agricoles et provenant de réserves instituées aux bornes d'une usine hydraulique reliée directement ou indirectement à la présente concession et de livrer soit indirectement au bénéficiaire de la réserve, soit au réseau qui l'alimentera, une quantité correspondante d'énergie.

La redevance due de ce chef au concessionnaire, sera fixée par le ministre des Travaux publics, après avis du Comité d'Electricilé, le concessionnaire entendu, compte tenu des conditions d'alimentation de la concession.

ART. 13. - Obligation de consentir des abonnements sur tout le parcours de la distribution. - Sur tout le parcours de la distribution, le concessionnaire sera tenu de fournir l'énergie électrique dans les conditions prévues au cahier des charges, à toute personne qui demandera à contracter un premier abonnement d'une durée d'au moins ......... années (3). Ceet

(1) La désignation sera faite par le préfet si la distribution s'étend sur le territoire d'un seul déparrtement et par le ministre des Travaux publics si elle s'étend sur plusieurs départements.

(2) Ce délai sera d'un an au maximum.

(3) La réduction sur le tarif maximum stipulce au profit des services publics de l'Etat, des départements et des communes, des abonnement pourra ensuite être renouvelé par période de....(1).

La lourniture de courant devra être assurée dans un délai de un mois, augmenté du temps normalement nécessaire à l'exéculion des travaux, à partir de la date à laquelle aura été présentée la demande accompagnée des autorisations des propriélaires.

En aucun cas, le concessionnaire ne pourra être astreint à dépasser pour l'ensemble de la distribution, la puissance maximum de....... kiloxwatts en basse tension et de ...... kilowatts en haute tension.

Si les demandes viennent à dépasser la puissance disponible, elles seront desservies dans l'ordre de leur inscription sur un registre spécial tenu à cet effet.

Le concessionnaire ne sera pas astreint à alimenter en basse tension les installations d'une puissance supérieure d̀ ...... kilowatts (1) ni à alimenter en haute tension les installations d'une puissance inférieure $\dot{a} \ldots . .$. kilowatts (2).

Art. 14. - Extension du réseau. - Le concessionnaire sera tenu d'établir toute canalisation ou tous ouvrages accessoires dont..... des frais de premier établissment seront payés par un ou plusieurs abonnés collectivement.

Le payement des frais correspondant aux canalisations sera remplacé si l'abonné (ou les abonnés) le demande (nt) par le versement pendant cinq années, d'une redevance d'établissement forfaitaire et annuel de $(3) \ldots \ldots \ldots \ldots \ldots$ par mètre de canalisation.

Cette redevance sera diminuée de ........ des recettes annuelles de courant ( $y$ compris le terme correctif), perçues sur les abonnés desservis par l'extension.

Un nouvel abonné ne peut être branché sur l'extension quà la condition suivant les cas :

$1^{\circ}$ De rembourser une part proportionnelle à la puissance prise et à la fraction des installations utilisées au transport de cette puissance, des charges de premier établissement supportées en capital par les abonnés antérieurs, ces charges étant toutefois diminuées d'autant de cinquième de leur valeur qu'il s'est écoulé d'années depuis l'établissement de l'extension.

$2^{\circ}$ De participer à la redevance d'établissement de l'extension, proportionnellement à la puissance prise, et à la partie des installations utilisées au transport de cette puissance.

Les projets de canalisations et ouvrages réclamés devront ètre présentés par le concessionnaire dans le délai de trois mois à partir de la demande qui lui aura été faite, accompagnée de l'engagement de satisfaire aux conditions prévues ci-dessus. La ligne devra ètre achevée et mise en service dans le délai de ....... mois (4), à dater de l'approbation des projets si sa longueur est inférieure à ........ mètres, et dans le délai de ....... mois, si sa longueur est supérieure.

\section{Le concessionnaire sera dispensé de l'obligation d'étendre le}

établissements publics et des associations syndicales ne peut ètre inférieure à 10 pour 100 en basse tension.

(1) Ces durées peuvent ètre différentes en haute tension et en basse tension.

(2) Cette clause n'a pas à ètre insérée dans les cahiers des charges des concessions comportant uniquement de la basse tension. La limitation en haute tension devra ètre inférieure à la limitation en basse tension.

(3) Cette redevance peut ètre différente suivant la tension de la ligne et les conditions techniques d'établissement, telles que dispositions en aérien ou souterrain, etc. Elle peut tenir compte de la situation économique lors de l'établissement de l'extension.

(4) En aucun cas, le premier délai ne doit dépasser six mois. 
rése'an si le's demandes dabonnement dépassent la puissance disponible sur le maximum prónu it larticle 13 (1).

Le concessiomnaire pourra, en outre, sons réserve de l'approbation des projets, établir dans le périmctre de la concession lous ouvrages et canalisations qu'il jugera utiles.

Les ouvrages et canalisalions élablis en vertu du présent article font partie intégranle de la distribulion.

ArT. 15. - Branchements (2). - Les branchements extéricurs ayant pour objet d'amener le courant du réseau à l'intérieur des propriétés desservies jusques et y compris, soit la boilte du coupe-circuit principal, soil le poste de transformation, seront installés el entretenus par le concessiomnaire et feront partie intégrante de la distribution. Les frais d'installation de ces branchements extérieurs seront remboursés au concessionnaire par les propriétaires ou abonnés, conformément au larif ci-après :

Les propriétaires ou abonnés qui garantiront une consommation d'au moins ........ kilowatts-heures par an pendant ........ années seront dispensés du remboursement des frais d'installation des branchements extérieurs, à condition d'y substituer le payement d'une redevance mensuelle conformément au tarif ci-après:

Lorsque cette redevance aura été payée pendant la période mentionnée ci-dessous, les frais d'installation du branchement extérieur seront considérés comme amortis.

Les frais d'installation des branchements extérieurs resteront entièrement à la charge du concessionnaire si le; propriétaires ou abonnés garantissent une consommation d'au moins ......... kilowatts-heures par an pendant ........ années.

Les branchements intérieurs, les colonnes montantes et toutes dérivations seront établis et entretenus par les soins et aux frais des propriétaires ou des abonnés.

Toutefois, si les propriétaires le requièrent, le concessionnaire sera tenu d'exécuter et d'entretenir lui-même ces installations moyennant une rémunération calculée conformément au tarij ci-après :

Les tarifs prévus au présent article seront revisables à toute époque par un accord entre l'autorité concédante et le concessionnaire.

Art. 16. - Compteurs (3). - Les compteurs servant à mesurer les quantités d'énergie livrées aux abonnés par le concessionnaire, seront d'un des types approuvés par le ministre des Travaux publics, après avis du Comité d'Electricité institué conformément à la loi du 15 juin 1906. Pour chaque type, le ministre déterminera la valeur des écarts dans la limite desquels les compteurs seront considérés comme exacts.

Les compteurs seront posés, plombés et entretenus par le concessionnaire.

(1) A insérer seulement lorsque la puissance à fournir par le concessionnaire est limitée par le cahier des charges.

(2) Les tarifs et garanties figurant à cet article peuvent différer suivant les caractéristiques des branchements.

Des réductions peuvent être prévues en faveur des abonnés qui souscrivent leur polices ou des engagements analogues avant une date déterminée.

(3) Les tarifs et garanties figurant à cet article peuvent différer suivant les caractéristiques des appareils.

Des réductions peuvent être prévues en faveur des abonnés qui souscrivent leurs polices ou des engagements analogues avant une date déterminée.

L'Etat peut spécifier que le fourniture du compteur sera toujours faite par le concessionnaire. Dans ce cas, les $2^{\mathrm{e}}, 3^{\mathrm{e}}, 4^{\mathrm{e}}, 5^{\mathrm{e}}$ et $6^{\mathrm{e}}$ alinéas de l'article 16 seront remplacés par un alinéa unique :

"Les compteurs seront fournis posés, plombés et entretenus par le concessionnaire qui percevra, à titre de rémunération pour ce service, une somme mensuelle de .....".
L'abomé anra la laculte de les l'ournir lui-même on de demander all concessionnaire de les fournir.

Le concessionnaire percevra à litre de frais de pose une somme de $\ldots \ldots \ldots$.

Si le compleur est fourni par le concessiomnaire, celui-ci percevra à litre de frais d'usage el d'entretien une somme mensuelle de........

Si lé compleur est fourni par l'abonné, le concessionnaire percevra, à titre de frais d'edtretien, une somme mensuelle de...

Les Larils prévus au présent arlicle seront revisables à toule époque par un accord entre l'autorité concédante et le concessiomaire.

ArT. 17. -... Vérification des Compteurs. -..- Le concessionnaire pourra procéder à la vérification des compleurs aussi souvent qu'il le jugera utile, sans que celle vérification donne lieu à son profit à aucune allocation en sus des frais d'entretien menlionnés à l'arlicle précédent.

L'abonné auma loujours le droil de demander la vérification du compteur, soil par le concessionnaire, soit par un expert désigné d'un commun accord ou, à défaut d'accord, désigné par l'ingénieur en chef du contrôle des distributions d'énergie électrique. Les frais de là vérification seront à la charge do l'abonné si le compteur est reconnu exact ou si le défaut d'exactitude est à son profit; ils seront à la charge du concessionnaire si le défaut d'exactitude est au détriment de l'abonné.

ArT. 18. -- Police d'abonnement. - Les contrals pour la fourniture de l'énergie électrique seront établis sous la forme de polices d'abonnement, conformes aux modèles arrètés d'accord entre le représentant de l'autorité concédante désigné par le ministre et le concessionnaire. Il ne pourra ètre dérogé aux dispositions contenues dans ces modèles que par une convention spéciale entre le concessionnaire et l'abonné, soumise aux conditions stipulées dans les deux derniers alinéas de l'arlicle 11 ci-dessus.

Dans le cas oì il y aurait lieu, au cours de la concession, d'apporter des modifications aux modèles de police, à défaut d'accord entre le représentant de l'autorité concédante désigné comme il est dit ci-dessus et le concessionnaire, il sera statué par le ministre des Travaux publics après avis du Comité d'électricilé.

Avances sur consommation. -..- L'abonné sera tenu sur la demande. du concessionnaire de lui verser une abance sur consommation lors de la signalure de la police; rette abance sera revisée, s'il y a lieu, lors du renouvellement de la police, soit par tacite reconduction, soit par tout autre mode.

Cette anance ne pourra être supérieure à la valeur de........ kilonalts-heures par hectonalt de puissance du compteur (comple tenu du terme correctif).

L'avance ne sera pas productrice d'intérèt el sera remboursable a l'expiration de l'abonnement.

Art. 19. - Surveillance des installations intérieures. -.. Le courant ne sera livré aux abonnés que s'ils se conforment, pour leurs installations intérieures, aux mesures qui leur seront imposées par le concessionnaire, avec l'approbation de l'ingénicur en chef du Contrôle, en vue soil d'empêcher les troubles dans l'exploitation, notamment les défauts d'isolement et la mise en marche ou l'arrêl brusque des moteurs électriques, soit d'empêcher l'usage illicite du courant, soit d'éviter une déperdilion exagérée d'énergic dans les branchements el colonnes montantes avant les compleurs.

Le concessionnaire sera autorisé, à cel effet, à vérifier, à toute époque, l'installation inlérieure de chaque abonné. 
Si l'installation est reconnue délectueuse, le concessionnaire pourra se refuser à continuer la foumilure du courant. En cas de désaccord sur les mesures à prendre en vue de faire disparaître toute cause de danger ou de trouble dans le fonctionnement général de la distribution, il sera statué par l'ingénieur en chef du Contrôle, sauf recours au ministre des Travaux publics, qui décidera après avis du Comilé d'Electricité.

En aucun cas, le concessionnaire n encourra de responsabilités à raison des défectuosilés des installations qui ne seront pas de son fait.

\section{ART. 20 (1). - Conditions particulières du service.}

ART. 21. - - Durée de la concession. - - La durée de la présente concession esl fixée à ........ années (2), elle commencera à courir à la date de son approbation définitive (3).

ART. 22. - Reprise des installations en fin de concession. - - A l'époque fixée pour l'expiration de la concession, l'Elat aura, moyemnant un préavis de trois ans, la faculté de se subroger aux droits du concessionnaire et de prendre possession de tous les immeubles el ouvrages de la distribution et de ses dépendances.

Si l'Elal use de cetle faculté, les usines, sous-stations el postes de transformaleurs el de coupure, le malériel électrique el mécanique, ainsi que les canalisations et branchements faisant partie de la concession lui seront remis gratuitement et il ne sera attribué d'indemnité au concessionnaire que pour la portion du coûl de ces installations qui sera considérée comme n'étant pas amortie. Cetle indemnité sera égale aux dépenses, dûment justifiées, supportees par le concessionmaire pour l'établissement de ceux des ouvrages ci-dessus énumérés subsistant en fin de coneession qui auront été régulièrement exécutés pendant les $n$ dernières années de la concession en ce qui concerne les constructions; dans les $n / 2$ dernières années de la concession en ce qui regarde l'appareillage, sauf déduction pour chaque ouvrage de $1 / n$ en ce qui a trait aux constructions et de $2 / n$ en ce qui touche lappareillage de la parlie de sa valeur supportée par le concessionnaire pour chaque année écoulée depuis son achèvement. Toutefois, si le concessionmaire justifie que, malgré sa bonnę gestion, il a été dans l'impossibilité d'effecluer, au cours de certaines années d'exploitation, des amortissements de $1 / n$ et $2 / n$ prévus ci-dessus, par suile d'insuffisance des produits d'exploitation de la concession, les déductions à faire pour les excercices correspondants ne s'élèveront qu'au montant des amortissements que le concessionnaire aura pu réellement opérer au moyen des produits de ces mèmes exercices.

Les dépenses de constructions comprennent outre les dépenses concernant les immeubles proprement dits, tous frais relatifs aux pylones et supports et aux lignes elles-mèmes; les dépenses d'appareillage comprennent les frais relatifs aux isolateurs, aux appareils de sécurité, de contrôle ou de mesure, à l'équipement électrique et à loutillage. L'indemnité sera payée au concessiomnaire dans les six mois qui suivront l'expiration de la concession. Cependant, si, trois mois avant la date d'expiration de la

(1) L'article 20 indique si l'énergie doit ètre à la disposition des abonnés en permanence ou si le service peut être normalement suspendu à des heures déterminées, qui peuvent ètre variables suivant les saisons.

Il peut contenir, en outre, des conditions spéciales qui seraient stipulées pour la fourniture de l'énergie à certaines eatégories d'abonnés.

(2) La durée ne peut être supérieure à quarante ans.

(3) Lorsque la concession a pour objet l'extension d'une concession déjà existante, elle doit prendre fin à la même date que la concession principale, et l'article 21 détermine la date d'expiralion pour l'ensemble du réseau. concession, le concessionnaire justifie de dettes, emprunts ou autres charges concernant la concession. il en sera dressé un état visé par l'ingénieur en chef et l'Etat sera tenu d'assurer, dans la limile du montant de l'indemnité à allouer au concessionnaire, le service de ces dettes, emprunts ou charges, à leur échéance.

En ce qui concerne le mobilier et les approvicionnements, l'Etat se réserve le droit de les reprendre en totalité ou pour telle partie qu'il jugera convenable, mais sans pouvoir y être contraint. La valeur des objets repris sera fixée à l'amiable ou à dire d'expert et payée au concessionnaire dans les six mois qui suivront leur remise à l'Etat.

Si l'Etat ne prend pas possession de la distribution, le concessionnaire sera lenu d'enlever, à ses frais et sans indemnité, toutes celles de ses installations qui se trouvent sur ou sous les voies publiques; uil pourra, toutefois, abandonner sans indemnité les canalisations souterraines, à condition qu'elles n'apportent aucune gêne aux services publics.

Dans tous les cas, l'Etat aura la faculté, sans qu'il en résulte un droit à indemnité pour le concessionnaire, de prendre, pendant les six derniers mois de la concession, toutes mesures utiles pour assurer la continuité de la distribution de l'énergie en fin de concession, en réduisant au minimum la gêne qui en résultera pour le concessionnaire. Il pourra, notamment, si les sous-stations et postes de transformateurs n'appartiennent pas en propre au concessionnaire ou si celui-ci ne produit pas le courant dans des usines faisant partie de la concession, desservir directement les abonnés par des sous-stations ou postes de transformateurs nouveaux, en percevant à son profit le prix de vente de l'énergie et, d'une manière générale, prendre toutes les mesures nécessaires pour effectuer le passage progressif de la concession ancienne à une concession ou à une entreprise nouvelle.

ARt. 23. - Rachat de la concession. - A l'expiration d'un délai de dix ans, à compter de la date de l'approbation de la concession, l'Etat aura le droit de racheter la concession entière movennant un préavis de deux ans.

Le concessionnaire aura le droit de choisir entre les deux modes suivants de payement de l'indemnité de rachat, sauf pendant les (1) ....... dernières années de la concession au cours desquelles le deuxième mode de rachat ne sera pas applicable.

A. - Premier mode de pauement. - Le concessionnaire recevra pour indemnité :

10 Pendant chacune des années restant à courir jusqu'à l'expiration de la concession, une annuité égale au produit net moyen des sept années d'exploitation précédent celle où le rachat sera effectué, déduction faite des deux plus mauvaises.

Le produit net de chaque année sera calculé en retranchant des recettes toutes les dépenses faites pour l'exploitation de la distribution $y$ compris l'entretien et le renouvellement des ouvrages et du matériel, ainsi que les sommes versées à titre de redevance, conformément aux indications de l'article 5, mais non compris les charges du capital ni l'amortissement des dépenses de premier établissement. Dans aucun cas, le montant de l'annuité ne sera inférieur au produit net de la dernière des sept années prises pour, terme de comparaison;

$2^{\circ}$ Une somme égale aux dépenses dûment justifiées supportées par le concessionnaire pour l'établissement de ceux des ouvrages de la concession subsistant au moment du rachat, non compris

(1) Ce nombre ne devra pas être inférieur à 10 ni supérieur à 15 . 
ceux définis par le premier alinéa de l'article 6 ci-dessus, qui auront été régulièrement exécutés, pendant les $p$ années précédent le rachat en ce qui concerne les constructions, pendant les $p / 2$ années en ce qui concerne l'appareillage, sauf déduction pour chaque ouvrage de $1 / p$ en ce qui concerne la construction et de $2 p$ en ce qui concerne l'appareillage de la partie de sa valeur supportée par le concessionnaire pour chaque année écoulée depuis son achèvement.

B. - Deuxième mode de payement. - Le concessiomaire recevra une indemnité égale aux dépenses utiles et justifićes de premier établissement, supportées par lui, y compris les frais de constitution de société, s'il y a lieu, dans la limite d'un maximum de

Dans le cas où le montant des insuffisances annuelles qui se seraient produites depuis l'origine de la concession dépasserait le montant des excédents annuels constatés pendant la mème durée, la différence serait ajoutée à l'indemnité ci-après :

$1^{0}$ Frais d'exploitation y compris les sommes versées conformément à l'article 5 , à titre de redevances;

$2^{\circ}$ Frais de renouvellement des ouvrages et du matériel;

$3^{0}$ Intérèt et amortissement des emprunts contractés pour l'établissement de la distribution, sans que le montant total des amortissements à admettre en compte pour le calcul des insuffisances, puisse dépasser un tiers du montant desdits emprunts ;

$4^{\circ}$ Intérèt, au taux déterminé, en ajoutant deux points au taux du revenu donné par la rente perpétiuelle française comportant l'intérêt nominal le plus élevé, d'après le cours moyen de cette rente pendant l'année considérée, des sommes fournies par le concessionnaire au moyen de ses propres ressources et de son capital actions.

Quel que soit le mode de payement adopté, l'Etat sera tenu de se substituer au concessionnaire pour l'exécution des traités d'abonnement en cours visés par l'article 18 ci-dessus, ainsi que des contrats d'achat d'énergie et des autres engagements prix par lui en vue d'assurer la marche normale de l'exploitation, et de reprendre les approvisionnements en magasin ou en cours de transport, ainsi que le mobilier de la distribution. La valeur des objets repris sera fixée à l'amiable ou à dire d'expert, et sera payée au consessionnaire dans les six mois qui suivront leur remise à l'Etat.

Art. 24. - Remise des ouvrages. - En cas de rachat, ou en cas de reprise à l'expiration de la concession, le concessionnaire sera tenu de remettre à l'Etat tous les ouvrages et le matériel de la distribution en bon état d'entretien.

L'Etat pourra retenir, s'il y a lieu, sur les indemnités dues au concessionnaire, les sommes nécessaires pour meltre en bon état toutes les installations.

Lorsque l'Etat usera de la faculté, à lui réservée, de reprendre les installations en fin de concession, il pourra se faire remettre les revenus de la distribution dans les deux dernières années qui précéderont le terme de la concession et les employer à rétablir en bon état les installations, si le concessionnaire ne se met pas en mesure de satisfaire pleinement et enlièrement à cette obligation et si le monlanl de l'indemnité à prévoir en raison de la reprise de la distribution par l'Etat, joint au cautionnement, n'est pas jugé suffisant pour couvrir les dépenses des travaux reconnus nécessaires.

Art. 25. -- Déchéance et mise en régie provisoire. - - Si le concessionnaire n'a pas présenté les projels d'exéculion, ou s'il n'a pas achevé et mis en service les lignes de distribution dans les délais et conditions fixés par le cahier des charges, il encourra la déchéance qui sera prononcée.après mise en demeure, par décel, saul recours au Conseil d'Elat par la voie contenlieuse.

Si la sécurite publique vient à être compromise, le préfet, après avis de l'ingénieur en chef du contrôle, prendra, aux frais et risques du concessionnaire, les mesures provisoires nécessaires pour prévenir toul danger. Il soumeltra au ministre des Travaux publies les mesures qu'il aura prises à cet effet. Le ministre prescrira, s'il y a lieu, les modifications à apporter a ces mesures ef adressera au concessiomaire une misc en demeure fixant le délai à lui imparli pour assurer à l'avenir la sécurité de l'exploitation.

Si l'exploitation vient à etre interrompue en partie on en totalité, il y sera également pourru aux frais el risques du concessionnaire. Le préfet soumeltra immedialement au ministre des Travaux publies les mesures qu'il comple prendre pour assurer provisoirement le service de la distribution. Le ministre statuera sur ces propositions ef adressera une mise en demeure fixant un délai au concessionnaire pour reprendre le service.

Si, à l'expiration du delai imparli, dans les cas prévus aux deux alinéas qui précèdent, il n’a pas été salisfail à la mise en demeure, la déchéance pourra être prononcée.

La déchéance pourra également ètre prononcée si le concessionnaire, après mise en demeure ne constitue pas le cautionnement prévu à l'article 31 ci-après, dans le cas où des prélèvements auraient été effectués sur ce cautionnement en conformité des dispositions du cahier des charges.

La déchéance ne serait pas encourue dans le cas où le concessionnaire n'aurait pu remplir ses obligations par suite de circonstances de force majeure dûment constatées.

Art. 26. -- Procédure en cas de déchéance. -... Dans le cas de déchéance, il sera pourvu tant à la continuation et à l'achèvement des travaux qu'à l'exécution des autres engagements du concessionnaire au moyen diune adjudication qui sera ouverte sur une mise à prix des projets, des terrains acquis, des ouvrages exécutés, du matériel et des approvisionnements.

Cette mise à prix sera fixée par le ministre des Travaux publics sur la proposition du préfel, après avis du conseil municipal, le concessionnaire entendu.

Nul ne sera admis à concourir à l'adjudication s'il n'a au préalable, été agréé par le ministre des Travaux publics, et s'il n'a fait, soit à la caisse des dépôts et consignations, soit à la trésorerie générale du département, un dépôt de garantie égal au montant du caulionnement prévu par le présent cahier des charges.

L'adjudication aura lieu suivant les formes indiquées aux articles $11,12,13,15$ el 16 de l'ordonnance royale du 10 mai.

L'adjudicalaire sera soumis aux clauses du présent cahier des charges el substitué aux droits et charges du concessiomaire évincé, qui recevra le prix de l'adjudication.

Si l'adjudication ouverte n'amène aucun résultal, une seconde adjudication sera tentée sans mise à prix après un délai de trois mois. Si cette seconde tentative reste également sans résultal, le concessionnaire sera définilivement déchu de tous droits; les ouvrages el le malériel de la distribution ainsi que les approvisionnements deviendront sans indemnité la propriété de l'Etat.

Ar'T. 27. -- Redevances. -- Les redevances pour l'occupation du domaine public national et détpartemental ne sont pas réglées par : e cahier des charges; elles sont fixées conformément aux dispositions du décret du 17 octobre 1907. modilié par les décrets du 7 septembre 1912, du 17 mai 1921 et du 12 juin 1927. 
Il en est de même des redevances pour l'occupation du domaine public communal, à moins que des accords spéciaux ne soient intervenus entre certaines communes el le concessionnaire, conformément à l'article 3 dudit décret.

AnT. 28. - Elats slatistiques el contrôle des recettes. -- Le concessionnaire sera tenu de remettre chaque annéeà l'ingénieur en en chef du Contrôle un compte rendu statistique de son exploilation.

Ce compte rendu sera élabli conformément au modèle arrêté par le ministre des Travaux publics, après avis du Comité d'Electricité el pourra être publié en tout ou en partie.

Pour les communes avec lesquelles des accords auront été passés conformément à l'article 27 ci-dessus, le concessionnaire devra, en outre, adresser à l'ingénieur en chef du Contrôle, dans le courant du premier trimestre de chaque année, l'état des recelles réalisées pendant l'année précédente.

L'ingénieur en chef aura le droit de contrôler ces états; à cet effet, les agents du contrôle dùment accrédités pourront se faire présenter toutes pièces de comptabilité nécessaires pour leur vérification.

ArT. 29. - Impôts et droits d'octrọi. - Tous les impôts établis par l'Etal, les départements ou les communes, y compris les impôts relatifs aux immeubles de la distribution, seront à la charge du concessionnaire.

Au cas où des impôts nouveaux relatifs à la vente, la production, le transport ou la consommation de lénergie électrique frapperaient le concessionnaire, ce dernier se réserve le droit de demander une augmentalion des tarifs maxima fixés par l'arlicle 11 ci-dessus.

Il sera statué sur cette demande comme il est indiqué à l'arlicle 11 en matière de revision des tarifs maxima de base.

ArT. 30. - Pénalités - Faute par le concessionnaire de remplir les obligations qui lui sont imposées par le présent cahier des charges, des pénalités pourront lui ètre infligées, sans préjudice s'il y a lieu, de dommages et intérêts envers les liers intéressés. Les pénalités seront prononcées au profit de l'Etat par le préfet, après avis de l'ingénieur en chef du Contrôle.

Les pénalités seront appliquées dans les conditions suivantes :

En cas d'interruption générale non justifiée du courant dans une commune au moins, pénalité calculée au prorata du temps sur la base de ........ fr. par heure d'interruption et par commune intéressée.

En cas de manquement aux obligations imposées par les articles $6,9,13,14$, et 28 du présent cahier des charges, et par chaque infraction, pénalité de ....... par journée indivisible, jusqu'à ce que l'infraction ait cessé (1).

ARт. 31. -.. Cautionnement. --- Avant la signature de l'acte de concession, le concessionnaire déposera, soit à la caisse des dépôts el consignations, soit à la trésorerie générale du département, une somme de ............. en numéraire ou en rente sur l'Etat, en obligations garanties par l'Etat ou en

(1) Les amendes prévues peuvent n'ètre pas les mèmes pour les infractions aux divers articles mentionnés dans ce paragraphe. bons du 'Trésor, dans les conditions prévues par les lois et règlements pour les cautionnements en matière de travaux publics.

La somme ainsi versée formera le cautionnement de l'entreprise.

Sur le cautionnement seront prélevés le montant des amendes stipulées à l'article 30, ainsi que les dépenses faites en raison des mesures prises aux frais du concessionnaire pour assurer la sécurité publique ou la reprise de l'exploitation en cas de suspension, conformément aux prescriptions du cahier des charges.

Toutes les fois qu'une somme quelc onque aura été prélevée sur le cautionnement, le concessionnaire devra le compléter à nouveau dans un délai de quinze jours, à dater de la mise en demeure qui lui sera adressée à cet effet.

La moitié du cautionnement sera restituée au concessionnaire après achèvement du réseau principal de distribution prévu à l'article 6 ci-dessus ; l'autre moitié lui sera restituée en fin de concession. Toutefois, en cas de déchéance, la partie non restituée au cautionnement restera définitivement acquise à l'Etat.

ArT. 32. - Agents du concessionnaire. - Les agents et gardes que le concessionnaire aura fait assermenter pour la surveillance et la police de la distribution et de ses dépendances seront porteurs d'un signe distinctif et seront munis d'un titre constatant leurs fonctions.

ArT. 33. - Emplois réservés. - En conformité des lois et règlements actuellement en vigueur, le concessionnaire devra réserver aux anciens militaires, à leurs veuves et à leurs orphelins remplissant les conditions prévues par ces lois et règlements, un certain nombre d'emplois. ainsi qu'il est indiqué au tableau annexé au présent cahier des charges; il se conformera, à cet effet, aux dispositions édictées pour l'application des lois dont il s'agit.

ART. 34. - Cession ou modification de la concession. - Toute cession partielle ou totale de la concession, tout changement de concessionnaire, ne pourront avoir lieu à peine de déchéance, qu'en vertu d'une autorisation donnée par le ......... (1).

ART. 35. - Jugement des contestations. - Les contestations qui s'élèveraient entre le concessionnaire et l'administration, au sujet de l'exécution et de linterprétation des clauses du présent cahier des charges, seront jugées par le conseil de préfecture interdépartemental de ............... sauf recours au Conseil d'Etat.

Art. 36. - Election de domicile. - Le concessionnaire derra faire élection de domicile à

Dans le cas où il ne l'aurait pas fait, toute notification ou signification à lui adressée sera valable lorsqu'elle sera faite à la préfecture $d \quad \ldots \ldots \ldots \ldots \ldots$.

ART. 37. - Frais d'enregistrement. - Les frais de timbre et d'enregistrement du présent cahier des charges et des conventions annexées seront supportés par le concessionnaire.

(1) L'autorisation sera délivrée par le préfet ou le ministre des Travaux publics suivant que l'acte de concession aura été passé par le préfet ou par le ministre des Travaux publics. 